\title{
Germanica
}

\section{Zur Repoetisierung der deutschsprachigen Dramatik am Beginn des 21. Jahrhunderts}

The Return of the Poetic Function in Contemporary German Drama

Le retour à la poéticité dans les drames modernes de langue allemande

\section{Von Špela Virant}

\section{(2)enEdition}

Journals

Édition électronique

URL : http://journals.openedition.org/germanica/2540

DOI : $10.4000 /$ germanica. 2540

ISSN : 2107-0784

Éditeur

Université de Lille

\section{Édition imprimée}

Date de publication : 30 juin 2014

Pagination : 11-23

ISBN : 9782913857339

ISSN : 0984-2632

Référence électronique

Von Špela Virant, «Zur Repoetisierung der deutschsprachigen Dramatik am Beginn des 21.

Jahrhunderts », Germanica [Online], 54 | 2014, Online erschienen am: 30 Juni 2017, abgerufen am 06 Oktober 2020. URL : http://journals.openedition.org/germanica/2540 ; DOI : https://doi.org/10.4000/ germanica. 2540 


\title{
Zur Repoetisierung der deutschsprachigen Dramatik am Beginn des 21. Jahrhunderts
}

\author{
Von Špela VIRANT \\ Universität Ljubljana \\ Übers Verallgemeinern. \\ Niemals richtig. Immer wichtig. \\ Erich KÄSTNER
}

Das Ende des Kalten Krieges mit den darauf folgenden sozialen, politischen und geopolitischen Veränderungen sorgte für ein bewegtes Ende eines Jahrhunderts, das nach der ersten, extrem turbulenten Hälfte für Dekaden in Stagnation zwischen den unterkühlten Fronten $\mathrm{zu}$ versinken drohte. Gleichzeitig wurde auch die deutschsprachige Literaturszene neu belebt, vornehmlich im Bereich des Romans, der Kurzgeschichte und in der Dramatik. Die Reflexion über diese neuen Erscheinungen setzte schon Ende der 1990er Jahre ein und führte im Bereich der Dramatik zu heftigen, bis heute nicht enden wollenden Diskussionen. Über die Antwort auf die Frage, warum gerade die Dramatik so umkämpft wird, lässt sich nur mutmaßen. Zum einen mag die Distribution und Rezeption der Dramatik über das Theater eine schnellere und deutlichere Positionierung der Beurteilungen und Bewertungen fördern, zum anderen mag eben diese Distributionsart ein Aufeinanderprallen unterschiedlicher Betrachtungsweisen und Disziplinen begünstigen. So ist die Gegenwartsdramatik zum Untersuchungsgegenstand der Theaterwissenschaft geworden, der Kulturwissenschaft und der Literaturwissenschaft, die sich dem Gegenstand wiederum von mindestens zwei Seiten nähert: von der Gattungstheorie her und der Literaturgeschichte mit den Fragen der 
Periodisierung. Noch spannender wird die Diskussion wegen des gegenwärtigen Methodenpluralismus und - Eklektizismus, der innerhalb der Disziplinen herrscht und in jeder seine Besonderheiten entwickelt hat.

Der vorliegende Artikel, der sich der Gegenwartsdramatik von Seiten der Literaturgeschichte zu nähern versucht, will zu dieser Diskussion auf zwei Ebenen beitragen. Im ersten Teil soll so knapp wie möglich auf einige Aspekte und Begriffe der bisher geleisteten Forschungsarbeiten kommentierend eingegangen werden, damit auf dieser Grundlage im zweiten Teil auf den im Titel angeführten, nicht unproblematischen Aspekt der Repoetisierung hingewiesen werden kann.

\section{Immer wichtig}

Die meisten der bisherigen Studien zur Gegenwartsdramatik, um nur Gerda Poschmann, Hans-Thies Lehmann, Birgit Haas und Nikolaus Frei zu nennen, da ihre Beiträge den Impuls zur Diskussion gegeben haben, bemühen sich um einen möglichst komplexen, synthetischen Ansatz, der auf Verallgemeinerung hinausläuft. Da die deutschsprachige Theaterproduktion und Theatertextproduktion der letzten drei Dekaden sehr umfangreich und heterogen ist, sind Verallgemeinerungen besonders problematisch, worauf bereits oft hingewiesen wurde. Andererseits sind sie aber auch unumgänglich, nicht nur, weil sich in deren Diskussion bestimmte Schwerpunkte und Fragestellungen erst herauskristallisieren, sondern auch weil partikuläre Einzelstudien, die sich z. B. dem Werk eines einzelnen Autors oder einem einzelnen Werk widmen, die besonderen Leistungen dieses Werks nur auf der Folie der herrschenden Tendenzen seiner Zeit, in der Unterscheidung von der Zeit davor und danach hervorgehoben werden können. Nun fehlt bei der Gegenwartsdramatik das Danach, was an sich noch nicht problematisch ist, da die Forschungsarbeit dazu meist als work in progress verstanden wird, die in der Zukunft, durch Distanz und zusätzliche Informationen Korrekturen erfahren wird. Zu Problemen kommt es an anderen Stellen: Erstens bei der Frage „Wann setzt das Danach ein“, also bei der Periodisierung; zweitens „Ist es besser oder schlechter“, also bei der Wertung; und drittens „Setzt es überhaupt ein“, also bei Versuchen, gattungstheoretische Fragen ahistorisch, historisch oder inkonsequent historisch zu erörtern. Vor allem aber scheiden sich die Geister an dem Glauben an die Funktionen und Möglichkeiten des Schreibens über Kunst. Ist es seine Aufgabe und gleichzeitig einzige Möglichkeit nur zu beschreiben, was in der Kunst stattfindet, oder kann es die weitere Entwicklung der Kunst beeinflussen, sie in die eine oder andere Richtung lenken? Oder ist es gar autoreflexiv, von der Kunst abgekoppelt, mit sich selbst beschäftigt und belastet? In gewissem Sinne wiederholt dieses Schreiben jene gegensätzlichen Positionen, 
die es im Beschriebenen entdeckt, also jene Eigenschaften, die es dem Gegenwartstheater und der Dramatik zuschreibt: Autoreflexivität, Abbildung und Engagement. Die Leistung dieses Schreibens ist, dass es diese unterschiedlichen Positionen herausgearbeitet hat und langsam deutlich gemacht, dass sie gar nicht so unvereinbar sind, wie es um das Jahr 2000 noch schien.

Während die Autoreflexivität vor allem im nicht mehr dramatischen Theatertext und im postdramatischen Theater verortet wird, werden die Abbildung der Lebenswelt und Engagement der Gegenseite zugerechnet, die mit Begriffen wie reliterarisiertes Theater, redramatisierter Theatertext, politisches Theater und Text sowie neuer Realismus gekennzeichnet wird. Ausführliche Studien haben inzwischen die Fronten etwas aufgelöst und gezeigt, dass von keiner klaren Opposition ausgegangen werden kann, da sowohl in einzelnen Werkgruppen, z. B. bei Texten zur Wende, wie von Dag Kemser und Emmanuel Béhague untersucht $^{1}$, wie auch im Werk eines Autors oder sogar in einzelnen Werken, wie Christine Laudahn am Beispiel der Texte von Roland Schimmelpfennig zeigt ${ }^{2}$, Merkmale des Postdramatischen wie auch des Dramatischen oder des Post-Postdramatischen zu beobachten sind. Ljubinka Petrović-Ziemer zeigt in ihrer ausführlichen Übersicht über die bisherige Forschung ${ }^{3}$, wie diese Begriffe - mit gewissen Korrekturen, die bereits geleistet oder von ihr vorgeschlagen wurden $-{ }^{4}$ durchaus parallel für die unterschiedlichen Analysen Anwendung finden können.

$\mathrm{Zu}$ den Begriffen, die oft unterschiedlich aufgefasst werden, gehört auch der Begriff der Selbstreferenzialität. An dieser Stelle lässt sich weder das Eigenleben des Begriffs ausführlich nachzeichnen noch der Begriff klären, es soll nur auf eine Differenz im Gebrauch des Begriffs Referenzialität hingewiesen werden, die wohl darauf zurückzuführen ist, ob er in Anlehnung an Roman Jakobsons Kommunikationsmodell oder an Ferdinand de Saussures Zeichenmodell verwendet wird, obwohl er immer den Bezug der Sprache zur außensprachlichen Realität impliziert. Roman Jakobson bestimmte die referentielle Funktion der Sprache als die auf den Kontext gerichtete, informationsvermittelnde Funktion im Unterschied zur poetischen Funktion, bei der die Sprache auf sich selbst, ihre eigene ästhetische Organisation gerichtet ist. Der Unterschied zwischen der poetischen Sprache und der Alltags- oder Gebrauchssprache ist graduell: Während in der ersten die poetische

1. - Dag Kemser, Zeitstücke zur deutschen Wiedervereinigung, Tübingen, 2006; Emmanuel Béhague, Le Théâtre dans le réel, Strasbourg, 2006.

2. - Christine Laudahn, Zwischen Postdramatik und Dramatik, Tübingen, 2012.

3. - Ljubinka Petrović-Ziemer, Mit Leib und Körper, Bielefeld, 2011.

4. - Z. B. ihr wertvoller Beitrag zur Klärung des Subjektbegriffs im Kontext der Postdramatik-Diskussion: vgl. ebd. S. 113. 
Funktion über die referentielle dominiert, ist es in der zweiten gerade umgekehrt. Die anderen Funktionen werden von der Dominante nicht gelöscht. Wird jedoch Jakobsons Gedanke weitergeführt in Richtung einer Verabsolutierung der poetischen Funktion für poetische Texte, so kann es zu einer apriorischen Ab- oder gar Entwertung all jener Texte führen, in denen zwar die poetische Funktion dominiert, die jedoch deklariert über sich hinaus weisen wollen, wie z. B. die engagierte oder die didaktische Literatur. Ferdinand de Saussure hingegen beschrieb die Abkoppelung des sprachlichen Zeichens vom Referenten als Bedingung für das Funktionieren der Sprache, da man sonst für jedes Ding ein eigenes Wort haben müsste. Es ist als solches nicht etwas, was zur Disposition stünde, sondern die Voraussetzung für das Funktionieren der Sprache als Zeichen- und Kommunikationssystem. Die Literatur des 20. Jahrhunderts lässt sich daraufhin untersuchen, ob von ihr diese Feststellung wahrgenommen, wie sie verstanden und wie darauf reagiert wird. So lässt sich eine manchmal resignative, manchmal verspielte Abkehr von der Beschreibung der Welt beobachten und eine Hinwendung zur Beschreibung der Vorstellungen von Welt oder zur Autoreflexion, also zur Beschäftigung mit der Sprache als dem Material, aus dem Literatur gemacht wird, und mit der Literaturtradition, die durch das bereits Gemachte die Möglichkeiten des Neuen einschränkt. Ein Umbruch in den dominierenden Reaktionen darauf wird oft mit dem Übergang von der Moderne in die Postmoderne markiert. Da das Postdramatische nach Lehmann mit dem Postmodernen verbunden wird, soll nebenbei bemerkt werden, dass der in den 1980er Jahren entbrannte Streit um die Postmoderne eigentlich nie ausgefochten, sondern eher nur beigelegt wurde. Ein relativer Konsens wurde im ästhetischen Bereich erreicht, in Bezug auf eine künstlerische Strömung, eine Art Ismus, also Postmodernismus ${ }^{5}$. Der Konsens bezieht sich jedoch mehr auf eine Auswahl von Werken, die als postmodern bezeichnet werden können, denn auf die Beschreibung der Eigenschaften, die diese Bezeichnung rechtfertigten.

Das Werk, das mit breitester Zustimmung als paradigmatisches Beispiel der postmodernen Literatur verstanden wird, ist Umberto Ecos Roman Der Name der Rose. In seiner Nachschrift zum „Namen der Rose" legt Eco in einprägsamen Bildern dar, wie gerade durch das Zitieren, durch Intertextualität und deklarierte Autoreflexivität ein Weg gesucht und gefunden wird, um über die Welt zu sprechen ${ }^{6}$. So kann Selbstreferenzialität nicht als konzeptuelle Entscheidung betrachtet

5. - Der Unterschied zwischen Postmoderne und Postmodernismus wurde in der slowenischen Literaturwissenschaft genauer ausgearbeitet, vgl. dazu Janko Kos: Postmodernizem. Ljubljana, 1995.

6. - Umberto Eco, Nachschrift zum „Namen der Rose“, München, 1984. 
werden, die eingesetzt oder nicht eingesetzt wird, Autoreflexion nicht als grundsätzliche Verweigerung des Weltbezugs verallgemeinert und diese Verweigerung nicht als konstitutive Eigenschaft postmoderner Literatur behauptet werden, von der sich der neue Realismus durch die Bemühung um den Weltbezug deutlich absetzte. Der Unterschied, der nicht zu leugnen ist, besteht jedoch sicherlich in den Mitteln und Strategien, die in diesen Bemühungen bedient werden.

Ein weiterer Begriff, der diese Unterschiede verdeutlichen sollte und auf den ersten Blick erhellend erscheint, ist der Begriff der Mimesis. Unter Beachtung dieses Begriffs können postdramatische Werke der anti-mimetischen Tradition zugerechnet werden. Auf den zweiten Blick eröffnen sich dadurch jedoch andere Probleme. Die Sprache selbst wirkt deutlich mimetisch gerade in onomatopoetischen Wörtern, also da, wo sie als Klangmaterial wirkt. Die Entsemantisierung der Sprache ist kein Garant für die Eliminierung der mimetischen Dimension. Gleichzeitig kann die Mimesis, greift man auf Aristoteles zurück, nicht als Gegensatz der Poesis verstanden werden. Die Nachahmung, die dem Menschen angeboren und eine Freude sei, stellt für Aristoteles die Grundlage für die Entstehung der Dichtkunst dar ${ }^{7}$. Die Nachahmung von Handlung ist zwar für Aristoteles das wichtigste Element der Tragödie, jedoch nur eines von sechs, die er bestimmt ${ }^{8}$. Die Nachahmung ist nicht Ziel und Zweck der Dichtkunst, sondern wird als Ausgangspunkt und Mittel zum Erreichen künstlerischer Wirkungen gewissermaßen degradiert. Die Nachahmung von Realität wird zum Mittel der Erschaffung neuer Realitäten, die zwar nicht den gleichen ontologischen Status haben wie die nachgeahmte, ihr jedoch gleichwertig sind.

Noch schwieriger ist der Umgang mit dem Begriff Dekonstruktion. Ohne auf die bereits viel diskutierte Problematik der Dekonstruktion als Methode für die Analyse literarischer Texte einzugehen, soll nur auf ein Problem hingewiesen werden. Wird der Begriff der Dekonstruktion nur dafür verwendet, um einerseits den dramatischen Text als sinnfreies Spiel der Signifikanten zu definieren, andererseits aber gleichzeitig die Sinnkonstruktion dem Rezipienten zugeschoben, so findet sich dieser in der paradoxen Situation, dass er zwar nun alle Freiheit der Interpretation hat, jedoch jede Interpretation, die über die Feststellung der Sinnfreiheit des Textes hinausginge, a priori als falsch und nicht als eine der unterschiedlichen möglichen Interpretationen gelten muss.

7. - Aristoteles, Poetik. Stuttgart 1982, S. 11.

8. - Ebd., S. 19-21. 


\title{
Niemals richtig
}

Eine Repoetisierung für die gesamte Dramatik am Beginn des 21 . Jahrhunderts zu behaupten, wäre nicht nur niemals richtig, sondern auch unmöglich. Jedoch auch die Behauptung, eine Repoetisierung sei das Kennzeichen einer bestimmten Tendenz im Rahmen der Gegenwartsdramatik nach der Jahrtausendwende, ist vor allem als heuristisches Mittel zu betrachten, das möglicherweise helfen kann, gewisse Qualitäten dieser Texte hervorzuheben, um in zukünftigen Studien weitere Differenzierungen vorzunehmen.

Im vorangegangenen Abschnitt wurde schon die Richtung angedeutet, wie im vorliegenden Artikel die Poetizität dieser Repoetisierung verstanden werden will, jedoch ist es an dieser Stelle notwendig, den Begriff von einigen anderen gängigen Bezeichnungen abzugrenzen. Was ist damit nicht gemeint? Gemeint ist nicht die „Reliterarisierung des Theaters", die sich vor allem auf die Rückkehr des Textes ins Theater bezieht; nicht der ,lyrisierte Theatertext“, da sich dieser Begriff vor allem auf die Auflösung des Dramatischen hin zum lyrischen Text bezieht; nicht das poetische Theater im Sinne des Symbolismus oder in Versen geschriebene Lesedramen. Poetizität soll, erstens, in Anlehnung an Jakobson vor allem als die Qualität jener Texte verstanden werden, in denen die poetische Funktion der Sprache dominiert und die mehrdeutig sind ${ }^{9}$. Die referentielle Funktion, die erhalten bleibt, verringert die Poetizität nicht, da sie gewissermaßen eine Vorbedingung der Mehrdeutigkeit darstellt, wie Jakobson es formulierte: „Der Vorrang der poetischen Funktion vor der referenziellen löscht den Gegenstandsbezug nicht aus, sondern macht ihn vieldeutig"10. Die Bedeutung der Mehrdeutigkeit als wichtigen Differenzkriteriums für literarische Texte fasst Neva Šlibar wie folgt zusammen:

\begin{abstract}
„Zum einen ermöglicht Mehrdeutigkeit, dass ein literarischer Text sowohl im Laufe der Zeit wie auch gleichzeitig von verschiedenen RezipientInnen unterschiedlich gelesen wird, zum anderen können dieselben RezipientInnen diesen diachron, d.h. in unterschiedlichen Lebensphasen und - umständen, verschieden realisieren, jedoch immer kohärent und für sie relevant" 11 .
\end{abstract}

Bei Theatertexten ist es gerade ihre Mehrdeutigkeit, die - gleichzeitig oder im Laufe der Zeit - unterschiedliche Inszenierungen ermög-

9. - Zur Poetizität der Texte von René Pollesch vgl. Johann Reisser: Archäologische Schnitte, kollidierende Wucherungen: Das post-bürgerliche Schauspiel des Selbst in René Polleschs Theater des Sagbaren. In: Artur Pełka / Stefan Tigges (Hg.), Das Drama nach dem Drama. Bielefeld 2011, S. 287-302, S. 295f.

10. - Roman Jakobson, Poetik. Frankfurt a. M., 1979, S. 111.

11. - Neva Šlibar, RundUM Literatur, Ljubljana, 2009, S. 136. 
licht, auch im Rahmen unterschiedlicher Inszenierungspraktiken, die von der Kritik und vom Publikum nicht notwendigerweise immer einheitlich im gleichen Maße als relevant, jedoch als kohärent bewertet werden. Umgekehrt lässt sich aus der Anzahl und Diversität der Inszenierungen eines Stücks auf die wahrgenommene Mehrdeutigkeit des Textes schließen. In Slowenien wurden z. B. in den vergangenen Jahren fünf Stücke von Roland Schimmelpfennig übersetzt und inszeniert - das ist mehr als von anderen GegenwartsautorInnen -, und zwar von fünf RegisseurInnen, deren Inszenierungen man, grob formuliert, zweimal als postdramatisch, einmal als realistisch und zweimal als eine Art Mischform bezeichnen könnte ${ }^{12}$. Das kann darauf hindeuten, dass Schimmelpfennigs Stücke gegenwärtig als mehrdeutig wahrgenommen werden, es besagt jedoch noch nicht, dass sie im Laufe der Zeit als solche bestehen bleiben.

Zweitens ist für die Poetizität der Gegenwartsdramatik ein weiteres Kriterium bedeutend, das auch zu den wichtigsten Differenzkriterien für literarische Texte gezählt wird, und zwar das der Fiktionalität, dessen Bestimmung auf die Poetik von Aristoteles zurückgreift, genauer auf seine Unterscheidung zwischen Geschichtsschreibung und Dichtung. Die Aufgabe des Dichters sei nicht das mitzuteilen, ,was wirklich geschehen ist, sondern vielmehr, was geschehen könnte, $d$. h. das nach den Regeln der Wahrscheinlichkeit oder Notwendigkeit Mögliche"13. Zur Poetizität sollte also auch das Erschaffen einer eigenen Welt mit eigener Referenzialität und Kausalität gehören, oder genauer, das Erschaffen wenigstens einer möglichen Welt. In der sogenannten postmodernen Literatur kommt es zur Problematisierung der Abgrenzung von Fiktionalität in zwei Richtungen. Einerseits zielt die Problematisierung in Richtung der Realität, deren auf den Konventionen des common sense basierende Zuverlässigkeit in Frage gestellt wird, andererseits in Richtung einer reflexiven Metaebene. So entstehen elaborierte Verschachtelungen unterschiedlicher Fiktionalitätsebenen wie bei dem Stück Die arabische Nacht von Schimmelpfennig ${ }^{14}$.

Ein drittes Merkmal der zeitgenössischen repoetisierten Dramatik lässt sich nicht auf Grundmerkmale von literarischen Texten im Allgemeinen zurückführen, es lässt sich jedoch bei den meisten kanonisierten literarischen Werken auffinden. Es geht dabei um ein gewisses

12. - Push up 1-3, R: Mateja Koležnik, Prešernovo gledališče, Kranj 2003; Die arabische Nacht, R: Diego de Brea, SNG, Nova Gorica 2003; Die Frau von früher, R: Martin Čičvák, SNG Drama, Ljubljana 2006; Vorher/Nachher, R: Sebastijan Horvat, E.P.I. center, Ljubljana, 2006; Der goldene Drache, R: Janusz Kica, Teatro Stabile Sloveno, Triest 2010

13. - Aristoteles, S. 29.

14. - Roland Schimmelpfennig, Die arabische Nacht. In: Ders. Die Frau von früher, Frankfurt a. M. 2004. 
$\mathrm{Ma} ß$ an Komplexität, die weder so groß ist, dass sie die Werke hermetisch elitär wirken ließe, noch so klein, dass die Werke als veristisch populär gelten könnten. Diese Komplexität lässt sich zwar auf der formalen Ebene verorten - wie z. B. auf der Ebene der Lexik, der Syntax, der rhetorischen Figuren und Tropen wie auch im Umgang mit gattungsspezifischen Normen -, die jedoch in Relation zur thematischen Komplexität steht, deren Wahrnehmung wiederum als historisch bedingt erscheint. Mit anderen Worten, die politischen und soziokulturellen Umstände der Rezeption haben Einfluss darauf, welche Themen, in welcher Form vermittelt, überhaupt als solche wahrgenommen werden können. Es geht also nicht nur darum, innovativ von der Norm abzuweichen, sondern auch darum, was unter welchen Umständen als Norm und was als innovative Abweichung wahrgenommen werden kann. Bevor diese These jedoch genauer ausgeführt und illustriert wird, soll auf eine weitere Dimension dieser Problematik hingewiesen werden.

Vereinfachend und verallgemeinernd lassen sich in der Literatur und der Literaturwissenschaft zwei Konzepte der Relation zwischen Literatur und Welt, zwischen literarischer und außerliterarischer Realität, unterscheiden, deren Differenzen besonders unter Berücksichtigung der Zeit deutlich werden. Das erste Konzept sieht die außerliterarische Realität als präexistent an. Die literarische Realität wird nachträglich, aus der bereits bestehenden nichtliterarischen Realität heraus erschaffen und kann auf die außerliterarische bezogen oder als von ihr unabhängig angesehen werden. Sie kann die Gegenwart widerspiegeln oder als Orakel die Zukunft prophezeien, die zwar noch nicht eingetroffen, doch im Verhältnis zur literarischen Realität immer präexistent ist. Die Literatur kann sich hier zwar auf die Welt beziehen, sie kann jedoch nicht in sie eingreifen oder sie verändern. Das zweite Konzept räumt hingegen die Möglichkeit einer präexistenten literarischen Realität ein, einer Literatur, die die außerliterarische Realität beeinflussen oder gar hervorbringen kann. Ihr wird die potentielle Fähigkeit zugestanden, in die Welt eingreifen und sie verändern zu können. Dieses Konzept unterliegt sehr unterschiedlichen literarischen Erscheinungen, z. B. den Zaubersprüchen, der didaktischen und der engagierten Literatur, aber auch einem ästhetizistischen Dandyismus, der für die Ästhetisierung des Lebens nach den Vorgaben der Kunst plädiert ${ }^{15}$.

Die beiden Konzepte stehen sich nicht unbedingt als Gegensätze gegenüber, keines leugnet die objektive Existenz der außerliterarischen Realität. Vor allem aber sagen sie noch nichts über ihre tatsächliche Einlösbarkeit. Sie können Mischformen bilden, vor allem in 
der Kombination, wo die Zukunft zwar nicht präexistent, jedoch als offen und unvorhersehbar gedacht wird. Umgekehrt sieht der staatlich verordnete sozialistische Realismus in der DDR nach dem Zweiten Weltkrieg die Zukunft zwar als vorherbestimmt in ihrer zwangsläufigen Entwicklung zum Kommunismus, der Literatur wird jedoch trotzdem die Aufgabe der Erziehung der Leser zum Sozialismus gestellt. Das doppelte Scheitern dieses Projekts beweist aber auch nicht, dass diese Literatur die Welt nicht beeinflusst hat, sondern nur, dass ihre Wirkung nicht vorhersehbar und nicht kontrollierbar ist, auch wenn die Kontrolle in Form von Zensur und vorgeschriebenen Literaturinterpretationen im Unterricht institutionalisiert ist.

Wenn auch der staatlich verordnete Realismus vor allem die Unkontrollierbarkeit der anvisierten Auswirkungen in der Welt verdeutlicht, so lässt er sich doch in eine Reihe von erneut auftauchenden realistischen Tendenzen in den Literaturen des 20. Jahrhunderts einordnen, die auffallend regelmäßig nach großen politischen und gesellschaftlichen Umbrüchen zu verzeichnen sind. Dazu gehört der sozialistische Realismus nach der Oktoberrevolution, die Neue Sachlichkeit nach dem Ersten Weltkrieg, diverse realistische Schreibweisen nach dem Zweiten Weltkrieg, aber auch der wieder einmal neue Realismus nach dem Ende des Kalten Kriegs. Daher die These, dass sich diese Tendenzen nicht nur durch das kunstinterne Prinzip der Innovation durch Abweichung von den zur Norm gewordenen Schreibweisen des Ästhetizismus, Modernismus oder Postmodernismus gebildet haben, sondern auch als Reaktion auf die herrschenden Umstände in einer Welt, die sich nach dem jeweiligen Konflikt völlig neu ordnen musste. Diese realistischen Schreibweisen sind stilistisch schon sehr weit entfernt vom historischen Realismus. Sie liefern kein getreues Abbild der Welt, wie sie ist, sondern entwerfen ein Bild der Welt, auf das man sich einigen kann. Sie bringen unterschiedliche nebeneinander bestehende Weltbilder auf einen kleinsten gemeinsamen Nenner und formulieren ein knappes Weltbild, das von einem möglichst breiten Publikum als der Realität entsprechend wahrgenommen werden kann, um so als Grundlage einer konsensuellen Realitätsstiftung zu dienen und weiteres Handeln zu ermöglichen. Der Gesellschaft sichert dieser Konsens einen Zusammenhalt, und dem Einzelnen dient er als Orientierungshilfe in einer unübersichtlich gewordenen Welt. Demgegenüber hatten die diversen Varianten des postdramatischen Theaters vielmehr die Aufgabe, ein monolithisches Weltbild aufzubrechen, das sich in der beharrlichen Stagnation des Kalten Kriegs verfestigt hatte, wo es schon unmöglich geworden war, sich vorzustellen, dass die Trennung in Ost und West einmal wird aufgehoben werden können. Die Pluralisierung und Diversifizierung im ästhetischen und im 
sozialen Raum hat die Veränderungen denkbar gemacht und vielleicht auch zu ihrer Durchführbarkeit beigetragen.

Wie Birgit Haas in ihrem Plädoyer für ein dramatisches Drama betont, ist die Zukunftsorientiertheit gerade für das Theater und die Dramatik besonders wichtig ${ }^{16}$. Durch die kollektive Rezeptionsweise der Dramatik im Theater kann und muss sie auch schneller auf die veränderten Umstände reagieren. Zudem hatten die politischen Veränderungen große Auswirkungen gerade in der Theaterlandschaft, wie David Ashley Hughes bemerkt: „Was nach 1990 geschah [...], war eineZeit enormer Verwirrung, da die deutschen Theatergruppen umstrukturiert, Repertoires überholt und die Funktionen des Nationaltheaters neu durchdacht werden mussten" 17 . Es lassen sich also drei Gründe für den neuen Realismus in der Dramatik der 1990er Jahre formulieren: Erstens, die Abweichung von der zur Norm gewordenen Postdramatik, zweitens, das jenseits der Sprache anvisierte Ziel einer konsensuellen Realitätsstiftung, und drittens, die Suche nach einer Schreibweise, die den bereits bekannten realistischen Schreibweisen ähnlich genug ist, um vom Publikum als etwas Vertrautes inmitten einer fremd gewordenen Gegenwart empfunden zu werden, gleichzeitig aber weicht sie von den traditionellen Realismen genug ab, um als modern und der neuen Zeit entsprechend angesehen zu werden. Damit einher geht auch eine geringere thematische Komplexität, da es erst einmal notwendig und das einzig mögliche ist, einzelne Ereignisse, wie z. B. die deutsche Wiedervereinigung, oder begrenzte Lebensbereiche, wie z. B. Familie und Arbeitswelt, zu reflektieren. Komplexere Zusammenhänge lassen sich erst nach dem Jahr 2000 literarisch darstellen oder zumindest als solche rezipieren, nachdem die deutsche Gesellschaft eine relative Stabilität erreicht hat. In den Romanen, die um das Jahr 2000 und danach erschienen sind, lässt sich diese Tendenz an der Flut der Familien- und Generationenromane ablesen, die durch individuelle Geschichten die kollektive Geschichte des 20. Jahrhunderts darstellen, um dadurch das Gepäck, das ins neue Jahrhundert mitgenommen wird, zu inventarisieren. John von Düffel hat mit seinem Roman Vom Wasser bereits früh dieses Modell für sein Schreiben und mit seiner Dramatisierung der Buddenbrooks für die Bühne entdeckt, wo es sich zwar nicht so gut etablieren konnte, aber doch das Bedürfnis nach größerer Komplexität der Theatertexte verdeutlichte.

16. - Vgl. Haas, S. 54ff.

17. - "What occurred after 1990 [...] did mark a period of tremendous confusion as German theatre companies had to be restructured, repertoires overhauled, and the function of national theatre urgently rethought." David Ashley Hughes, Notes on the German Theatre Crisis, in : The Drama Review, vol. 51, n44 (Winter 2007), S. 133-155, S. 133. 
Die erhöhte Komplexität zeigt sich schon auf der Ebene der sprachlichen Gestaltung, die sich von der provokativ vulgären Sprache, die in den 1990er Jahren einige Texte auszeichnet, absetzt und in die der Umgangssprache nachempfundenen Dialoge auch Metaphern einbaut, ohne dass die Texte dadurch ihren dramatischen Charakter verlören. Theresia Walser lässt ihr Stück King Kongs Töchter mit der Metapher eines ,friedlichen Stalingrads“ 18 enden. In ihrem Stück Herrenbestatter z. B. lässt sie den Verkäufer Ellenbeck über das Wetter grübeln: „Es gibt Gipfeltage und Tage, denen sieht man schon morgens auf den Grund“.

In Roland Schimmelpfennigs Stück Die Frau von früher und Ulrike Syhas Privatleben entsteht Mehrdeutigkeit vor allem auf der Ebene des Aufbaus und lässt unter anderen möglichen Interpretationen eine Lesart zu, nach der sich in beiden Stücken gleich drei Themenkomplexe miteinander verschränken: Liebe, Ökonomie und Zeit. In beiden Stücken wird eine frühere Liebesbeziehung gezeigt, die als eine Art Kreditanstalt funktioniert und scheitert. Die Liebe wird dabei als eine Art Investition in die Zukunft gesehen, sie baut auf Dauer und Vertrauen, also auf der Kreditwürdigkeit der Partner. Die Grundlage für das Zustandekommen einer solchen Anstalt ist das lineare Zeitmodell, das die Rückzahlung des Kredits in der Zukunft verheißt, diese Zukunft jedoch auch gleichzeitig immer in utopische Ferne entrücken lässt. Verlangt einer der Partner die sofortige Rückzahlung des Kredits, droht der Bankrott. In beiden Stücken sind es Frauen, die Forderungen nach der sofortigen Einlösung von Versprechen stellen, die von den Männern für die Zukunft gegeben worden sind. Beide Frauen sind nicht kompromissbereit und lassen sich nicht zum Schuldenerlass überreden. Doch während es bei Syha nur zu einer alltäglich unschönen Trennung kommt, die Raum für eine neue, bessere Beziehung schafft, endet die Geschichte bei Schimmelpfennig in einer Katastrophe mit Mord, Totschlag und Feuersbrunst.

Doch beide Stücke begnügen sich nicht mit der Diskussion über Vertrauensverlust und Schuldenerlass. Sie problematisieren die Grundlage für dieses Beziehungsmodell, indem sie die auf der Bühne dargestellte Zeit nicht als linear und kontinuierlich ablaufend erscheinen lassen. Syha beginnt und endet ihr Stück mit einer Variation der gleichen Szene, dazwischen wird die Geschichte in Zeitsprüngen erzählt, wobei die Vergangenheit immer als Gegenwart neben der eigentlichen Gegenwart dargestellt wird und die verschiedenen Zeitebenen ineinander greifen. Noch radikaler ist Schimmelpfennigs Kritik am gängigen Zeitmodell, da er nicht wie Syha auf zirkuläre Modelle zurückgreift, sondern mit Brüchen und Wiederholungen arbeitet, wodurch Kontinuität

18. - Theresia Walser, King Kongs Töchter, in: Theater heute, 11, 1998, S. 89. 
und Irreversibilität der Zeit in Frage gestellt werden. Zwar lassen sich in beiden Stücken lineare, zusammenhängende Geschichten konstruieren, doch schwingt das Wissen um ihre Konstruiertheit immer mit. Beide Autoren verbinden so die Krise der Liebesbeziehungen mit den ihnen unterliegenden Zeitvorstellungen. Doch bei Syhas Stück wird das Problem in dem vormodernen zirkulären Zeitmodell verortet, das mit, wie sie es nennt, ,zyklischen Verhaltensmustern“ verbunden ist ${ }^{19}$. Die Lösung, die suggeriert wird, ist das Aufbrechen dieser Verhaltensmuster und die Restitution des linearen Zeitmodells, in dessen Rahmen neue Investitionen in einen nun hoffentlich vertrauens- und kreditwürdigen Partner getätigt werden können. Bei Schimmelpfennig gibt es jedoch weder einen Rückgriff auf vormoderne Zeitvorstellungen noch den Glauben an eine mögliche Restitution des neuzeitlichen Zeitmodells. Sein Zerfall ist unabwendbar. Ohne ihn kann das Konzept der Liebesbeziehung als Kreditanstalt nicht mehr bestehen, aber einen Ausweg, ein neues, alternatives Konzept bietet auch Schimmelpfennig nicht an. Am Ende des Stücks hat die Zukunft keine Zukunft, alle Türen sind verschlossen und der Brand ist bereits entfacht.

Walsers, Schimmelpfennigs und Syhas Texte sind Beispiele dafür, wie in der zeitgenössischen Dramatik Mehrdeutigkeit auf unterschiedlichen Ebenen hergestellt wird, die zur Poetizität dieser Texte beiträgt, wodurch unterschiedliche Interpretationen, Inszenierungen und nicht zuletzt auch Übersetzungen möglich werden und zum gegenwärtigen Erfolg der Stücke beitragen.

\section{Literatur}

Aristoteles: Poetik, Stuttgart, 1982.

Béhague, Emmanuel: Le Théâtre dans le réel, Strasbourg, 2006.

Eco, Umberto: Nachschrift zum „Namen der Rose“, München, 1984.

Frei, Nikolaus: Die Rückkehr der Helden, Tübingen, 2006.

Haas, Birgit: Plädoyer für ein dramatisches Drama, Wien, 2007.

Hughes, David Ashley: Notes on the German Theatre Crisis. In: The Drama Review, vol. 51, n4 (Winter 2007), S. 133-155.

Jakobson, Roman: Poetik. Frankfurt a. M., 1979.

Kemser, Dag: Zeitstücke zur deutschen Wiedervereinigung, Tübingen, 2006.

Kos, Janko: Postmodernizem, Ljubljana, 1995.

Laudahn, Christine: Zwischen Postdramatik und Dramatik, Tübingen, 2012.

Lehmann, Hans-Thies: Postdramatisches Theater, Frankfurt a. M., 1999.

19. - Ulrike Syha, Privatleben. In: Theater heute, 12, 2008, S. 2. 
Pełka, Artur / Tigges, Stefan (Hg.): Das Drama nach dem Drama, Bielefeld, 2011.

Petrović-Ziemer, Ljubinka: Mit Leib und Körper, Bielefeld, 2011.

Poschmann, Gerda: Der nicht mehr dramatische Theatertext, Tübingen, 1997.

Syha, Ulrike: Privatleben. In: Theater heute, 12, 2008.

Schimmelpfennig, Roland: Die Frau von früher. Frankfurt a. M., 2004.

Šlibar, Neva: RundUM Literatur I, Ljubljana, 2009.

Walser, Theresia: Herrenbestatter. In: Theater heute, 2, 2010.

Walser, Theresia: King Kongs Töchter. In: Theater heute, 11, 1998. 
